\title{
Multiple Giant Tarlov Cysts Presenting as Polyradiculopathy: A Rare Manifestation
}

\author{
Ahmed K ${ }^{1}$, Rauniyar RK ${ }^{1}$, Sah PL ${ }^{1}$, Dhungel K ${ }^{1}$, Gupta MK ${ }^{1}$, Ansari $S^{1}$ \\ ${ }^{1}$ Department of Radiodiagnosis and Imaging, B P Koirala Institute of Health Sciences, \\ Dharan, Nepal
}

\begin{abstract}
We describe a case of multiple giant Tarlov cysts presenting with complaints of low back pain with neurological claudication. Tarlov cysts are nerve root cysts found most commonly in the sacral roots, arising between the covering layer of the perineurium and the endoneurium near the dorsal root ganglion. Most of them are asymptomatic, usually detected as incidental findings on MRI. Symptomatic Tarlov cysts are extremely rare, commonly presenting as sacral or lumbar pain syndromes, sciatica or rarely as cauda equina syndrome. Tarlov cysts should be considered in the differential diagnosis of patients presenting with these complaints.
\end{abstract}

Keywords: Low back pain, Sacral perineural cyst, Sciatica, Tarlov cyst

\section{Introduction}

Tarlov's cysts (perineural cysts) are fluidfilled meningeal dilations of the posterior nerve root sheath, usually at the dorsal root ganglion. They are commonly viewed in the sacrum 1 but can also be observed in the lumbar, thoracic, and cervical spine. Isadore M. Tarlov first published his studies on Tarlov cyst in 1938 as an incidental finding at autopsy. ${ }^{1}$ Since then few cases have been reported in the literature., ${ }^{2,3,4}$ Paulsen reported the incidence of Tarlov cysts as $4.6 \%$ in patients with back pain and only $1 \%$ of them were symptomatic. ${ }^{4}$ Clinically it may present as low back pain, sciatica, coccydynia or cauda equina syndrome. The cysts are usually diagnosed

Correspondence to: Dr. Kaleem Ahmed, Associate Professor, Department of Radiodiagnosis and Imaging, B P Koirala Institute of Health Sciences, Dharan, Nepal. Email: drkalim17@yahoo.co.in on MRI, which reveals the lesion arising from the sacral nerve root near the dorsal root ganglion. ${ }^{5}$ Tarlov advised extensive surgery with sacral laminectomy and excision of the cyst along with the nerve root. $^{6}$ Paulsen reported CT-guided Percutaneous aspiration of these perineural cysts for relief of sciatica. ${ }^{4}$ Recently, microsurgical excision of the cyst has been advocated, combined with duraplasty or plication of the cyst wall. ${ }^{7}$ We report a case of symptomatic multiple giant Tarlov cysts presenting as polyradiculopathy which is a rare manifestation.

\section{Case Presentation}

A 29-year-old female presented with right thigh pain off and on for nine months. The pain was not associated with specific time, posture or activity and it used to get relieved by non steroidal antiinflammatory drugs (NSAID). Clinical examination at this stage did not reveal any findings at spine, bilateral 
hips and left thigh. For last three months, the intensity and duration of pain had increased, which was now not relieved by taking NSAID. The pain had progressed to the lower back and bilateral upper thigh up to the ankle. The pain was aggravated by activity and prolonged standing and was more bothersome in the evening. She used to get up in the middle of the night with pain. Later the patient started having rest pain as well. Straight leg raising was $50^{\circ}$ on the right side and normal on the left side.
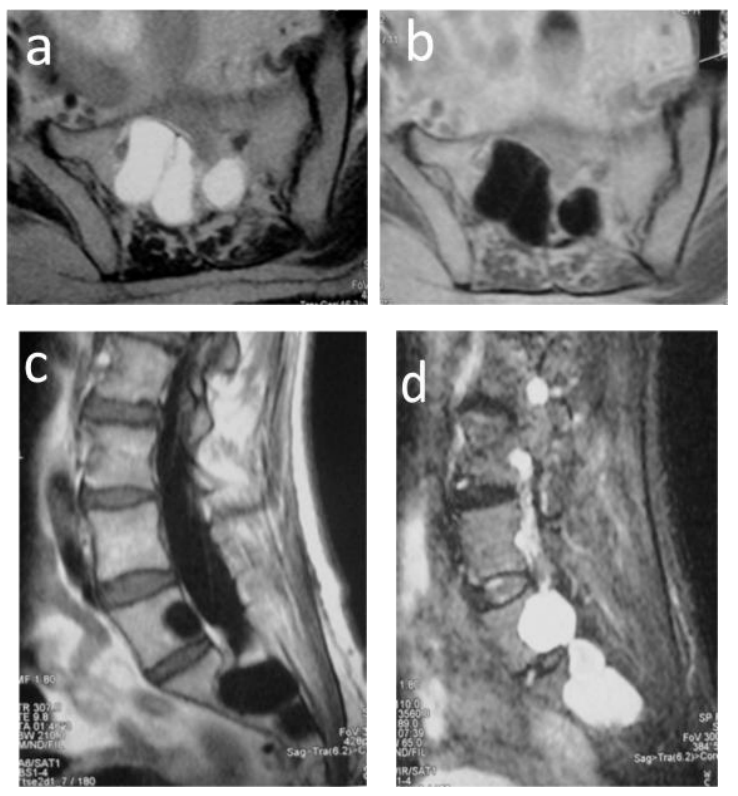

Fig 1 (a, b, c \& d): MRI of the lumbosacral spine (in axial plane - figures 1a \&b and in sagittal plane - figures 1 c \& d) revealed multiple, well circumscribed, fluid-filled cystic lesions arising from L5 toS4 vertebral levels, largest one measuring $3.5 \times 2.5 \mathrm{~cm}$ with widening of spinal canal, widening of neural foramina and scalloping of adjacent vertebral bodies. The lesions were hypointense on T1W and hyperintense on T2W \& STIR images.

Plain radiograph of the lumbosacral spine revealed scalloping of the vertebral bodies in lower lumbar and sacral regions. MRI of the lumbo-sacral spine (Figures 1a,1b,1c \& 1d in axial and sagittal plane) revealed multiple, well circumscribed, fluid-filled cystic lesions arising from L5 toS4 vertebral levels, largest one measuring $3.5 \times 2.5 \mathrm{~cm}$ with widening of spinal canal, widening of neural foramina and scalloping of adjacent vertebral bodies. The lesions were hypointense on $\mathrm{T} 1 \mathrm{~W}$ and hyperintense on T2W \& STIR images. CT scan of the lumbo-sacral spine (Figure $2 \mathrm{a} \&$ $2 b-$ in axial section with sagittal reconstruction) revealed cystic lesions with widening of spinal canal and scalloping of the adjacent vertebral bodies from L5 to S4 vertebral levels. The patient was taken for sacral laminectomy, excision of the cyst and plication of the cyst wall, while retaining the nerve root. Histopathological examination of the cyst wall showed nerve cells, which confirmed the diagnosis of Tarlov cyst. Postoperative period was uneventful and the patient made prompt recovery. Patient appreciated relief of pain immediately after the surgery. On nine months follow up, the patient had no pain in lower limbs and back and did not show any evidence of recurrence.

\section{Discussion}

Tarlov cysts are rare cause of low back pain and are more commonly seen in females. ${ }^{4,7}$ Clinical presentation of Tarlov cysts is variable. They may cause local and/or radicular pain and is referable to the caudal nerve roots, either sciatica, sacral or buttocks pain, vaginal or penile paraesthesia or sensory changes over the buttocks, perineal area and lower extremity. Depending on their location, size and relationship to the nerve roots, they may cause sensory disturbances or motor deficits. Tenderness on firm pressure over the sacrum may be present. Commonly, the symptoms are intermittent at its onset and are most frequently exacerbated by standing, walking and coughing. Bed rest alleviates the discomfort. $^{8}$

Plain X-rays are usually normal; however they may reveal bone erosion of the spinal canal or anterior or posterior neural foramina. $^{9}$ CT scan can demonstrate cystic 
masses isodense with CSF located at the foramina. ${ }^{10}$ MRI gives a much better soft tissue contrast and is currently the investigation of choice for perineural cysts. The cysts are hypointense on T1WIand and hyperintense on T2WI, similar to CSF. ${ }^{5}$ Myelography showing the filling of the meningocele sac $1 \mathrm{hr}$ after injection of contrast medium is highly suggestive of a perineural cyst. ${ }^{11}$

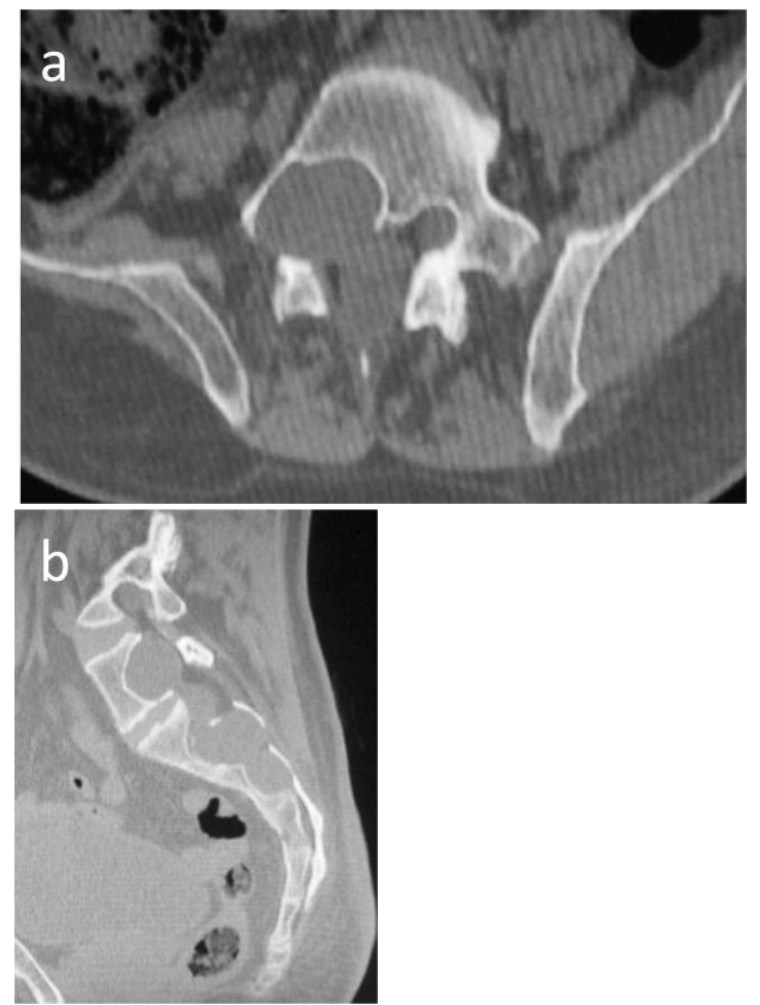

Fig 2a \& b: CT scan of the lumbo-sacral spine (in axial section - figure $2 a$, with sagittal reconstruction - figure $2 b$ ) revealed cystic lesions with widening of spinal canal and scalloping of the adjacent vertebral bodies from $\mathrm{L5}$ to $\mathrm{S} 4$ vertebral levels.

Microscopic features of the cyst were described by Tarlov. The early stage in cyst formation is that of a space between the arachnoid which covers the perineurium and the outer layer of the pial cover of the endoneurium. It usually begins in one portion of the circumference of the perineural space, the larger cysts compressing the nerve root to one side. The cyst occupies the posterior root abutting the proximal portion of the dorsal ganglion. Its main part is bordered by reticulum or by nerve fibers. ${ }^{1}$

The pathogenesis of perineural cysts is uncertain. According to Tarlov, hemorrhage into the subarachnoid space caused accumulations of red cells which impeded the drainage of the veins in the perineurium and epineurium, leading to rupture with subsequent cyst formation. Four out of the seven patients in Tarlov's 1970 article had a history of trauma. ${ }^{8}$ Schreiber and Haddad also supported this posttraumatic cause of cyst formation. ${ }^{12}$ Because many of the patients with perineural cyst in their series did not have histories of trauma, Fortuna et al. believed that the perineural cysts were congenital, caused by arachnoidal proliferations within the root sleeve. ${ }^{13}$

Tarlov advised that symptomatic, single perineural cysts should be completely excised together with the posterior root and ganglion from which they arise. ${ }^{8}$ Paulsen reported CT- guided percutaneous aspiration of perineural cysts for the relief of sciatica caused by compression. ${ }^{4}$ According to Caspar microsurgical excision of the cyst along with duraplasty or plication of the cyst wall is an effective and safe treatment of symptomatic sacral cysts and the parent nerve root is always left intact. ${ }^{7}$

Tarlov cysts are a documented cause of sacral radiculopathy and other radicular pain syndromes. They must be considered in the differential diagnosis of patients presenting with these clinical presentations and appropriately treated by cyst excision.

\section{References}

1. Tarlov IM. Perineural cysts of the spinal nerve roots. Arch Neural Psychiatry 1938; 40:1067-74.

2. Chaiyabud P, Suwanpratheep K. Symptomatic Tarlov cyst: Report and 
review. J Med Assoc Thai 2006; 89:1047-50.

3. Acosta FL Jr, Quinones-Hinojosa A, Schmidt MH, Weinstein PR. Diagnosis and management of sacral Tarlov cysts. Case report and review of the literature. Neurosurg Focus 2003;15:E15.

4. Paulsen RD, Call GA, Murtagh FR. Prevalence and percutaneous drainage of cysts of the sacral nerve root sheath (Tarlov cysts). AJNR Am J Neuroradiol 1994;15:293-9.

5. Rodziewicz GS, Kaufman B, Spetzler RF. Diagnosis of sacral perineural cysts by nuclear magnetic resonance. Surg Neurol 1984; 22:50-2.

6. Tarlov IM. Cysts (perineurial) of the sacral roots. J Am Med Assoc 1948; 138:740-4.

7. Caspar W, Papavero L, Nabhan A, Loew C, Ahlhelm F. Microsurgical excision of symptomatic sacral perineurial cysts: A study of 15 cases. Surg Neurol 2003;59:101-6.
8. Tarlov IM. Spinal perineurial and meningeal cysts. J Neural Neurosurg Psychiatry 1970;33:833-43.

9. Taveras JM, Wood EH. Diagnostic neuroradiology. $2^{\text {nd }}$ ed. Vol 2. Williams and Wilkins: Baltimore; 1976. p. 113945.

10. Tabas JH, Deeb ZL. Diagnosis of sacral perineural cysts by computed tomography. J Comput Tomogr 1986; 10:255-9.

11. Nishiura I, Koyama T, Handa $J$. Intrasacral perineurial cyst. Surg Neurol 1985;23:265-9.

12. Schreiber F, Haddad B. Lumbar and sacral cysts causing pain. J Neurosurg 1951;8:504-9.

13. Fortuna A, La Torre E, Ciappetta P. Arachnoid diverticula: A unitary approach to spinal cysts communicating with the subarachnoid space. Acta Neurochir (Wien) 1977;39:259-68. 